\title{
A Compression Original Image Based On The DDWT Technique And Enhancement SNR
}

\author{
$I^{*}$ A. K. Nahar, and ${ }^{1}$ Ansam S. Jaddar \\ ${ }^{1}$ University of Technology, Department of Electrical Engineering, Baghdad, \\ Iraq \\ *Email: alikareemnahar79@gmail.com
}

\begin{abstract}
Generally, Discrete wavelet transform (DWT) are good perform a when little to no simple mathematical operations in the wavelet basis, in many applications, wavelet transforms can be severely truncated compressed and retain useful information Image compression. Though, DWT and the divided wavelet transform, still suffering from Poor directionality Lack of phase information, and Shiftsensitivity, which is a major drawback in most the communications systems. The Double-Density Discrete Wavelet Transform (DDDWT) achieves great results compared to previous conventional methods less complexity. Credited with this good result, so due to a simplified account that deal with two-dimensional and three-dimensional images by the way and transformation matrices as if through a matrix multiplication between the picture and the conversion of number DDWT. Moreover, the form of repeated goal is achieved with the optimization process for the appropriate application.
\end{abstract}

Indexed Terms- DDDWT, Image Compression, Improve Medical Image.

\section{INTRODUCTION}

Data compression is the process of converting data files into smaller files for the efficiency of storage and transmission. As one of the enabling technologies of the multimedia revolution, data compression is a key to rapid progress being made in information technology. It would not be practical to put images, audio, and video alone on websites without compression [1]. Several benefits Image Compression and de-noising medical images emerging it as an attractive standard for various digital data over for various digital data over daily life applications [2]. Attention in ways that digital image stems from two main applications first one, improving the pictorial information for human interpretation of the areas of treatment; and as second, processing the image data to store and transport independent representation and visualization device. In some image processing applications require the transformation process to solve problems [3].

Although increasing the bandwidth is a possible solution, the relatively high cost makes this less attractive. Therefore, compression is a necessary and essential method for creating image files with manageable and transmittable sizes [4]. In order to be useful, a compression algorithm has a corresponding decompression algorithm that, given the compressed file, reproduces the original file. There have been many types of compression algorithms developed. These algorithms fall into two broad types, lossless algorithms and lessee algorithms. A lossless algorithm reproduces the original exactly. A lessee algorithm, as its name implies, loses some data. Data loss may be unacceptable in many applications. For example, text compression must be lossless because a very small difference can result in statements with totally different meanings [5]. There are also many situations where loss may be either unnoticeable or acceptable. In image compression, for example, the exact reconstructed value of each sample of the image is not necessary [6]. Depending on the quality required of the reconstructed image, varying amounts of loss of information can be accepted several methods are compared to their facility to compress standard images and the fidelity of the reproduced image to the original image. In 
order to compare wavelet methods, a software tool called Min-Image was used. Min-Image was originally created to test one type of wavelet [7].

However, the standard of DWT is an authoritative tool for analysis and processing of many realworld signals and images. These disadvantages severely restrict its scope for certain signal and image processing applications, for example, edge detection, image registration/segmentation, motion estimation [8]. Other extensions of standard DWT such as Wavelet Packet Transform (WPT) and Stationary Wavelet Transform (SWT) reduce only the first disadvantage of shift- sensitivity but with the cost of very high redundancy and involved computation. Recent research suggests the possibility of reducing two or more of these disadvantages. Introducing the DDWT as the tight frame equivalent of Daubechies orthonormal wavelet transforms [9]; the wavelet filters are of a minimal length and satisfy certain important polynomial properties in an over-sampled framework. For the reason that the DDWT, at each scale, has twice as many wavelets as the DWT, it achieves lower shift sensitivity than the DWT as in [10].

\section{THEORETICAL BACKGROUND}

\subsection{Characteristics of Discrete Wavelets Transform}

The DWT has the disadvantages of redundancy and impracticability with digital computers. As parameters $(a, b)$ take continuous values, the resulting DWT is a very redundant representation, and impracticability is the result of redundancy. So, the scale and shift parameters are evaluated on a discrete grid of time-scale plane leading to a discrete set of continuous basis functions. The discretization is performed by setting [11]:

$$
b=b_{0}^{j}, a=k b_{0}^{j} a_{0}, \text { for } j, k \in Z .
$$

Where $a 0>1$ is a dilated step and $b 0 \neq 0$ is a translation step. The family of wavelets then becomes:

$$
\psi_{j, k}(t)=a_{0}^{-j / 2} \psi\left(a_{0}^{-j} t-k b_{0}\right)
$$

And the wavelet decomposition of a function $f(t)$ is:

$$
f(t)=\sum_{j=0}^{\infty} \sum_{k=-\infty}^{\infty} D_{f}(j, k) \psi_{j, k}(t)
$$

Where a 2-D set of coefficients DF $(j, k)$ is called DWT of a given function $f(t)$. The selection of $\psi(t)$ is made in such a way that basis function set $\{\psi \mathrm{j}, \mathrm{k}\}$ constitute an orthonormal basis of $\mathrm{L} 2(\mathrm{R})$ so that:

$$
D_{f}(j, k)=\int \psi_{j, k}^{*}(t) f(t) d(t)=\left\langle\psi_{j, k}(t) f(t)\right\rangle
$$

\subsection{Computation technique of the DDWT}

The DDWT is implemented on discrete-time signals using the over-sampled analysis and synthesis filter bank shown in Figure (1). The up-sampled signals are filtered by the corresponding synthesis low pass $h 0(n)$ and two high pass $h 1(n)$ and $h 2(n)$ filters and then added to reconstruct the original signal. Note that the filters in the synthesis stage, are not necessarily the same as those in the analysis stage. For an orthogonal filter bank, $h i(n)$ has been just the time reversals of $h i(n)$. 
Wavelet frames, having the form described above, have twice as many wavelets than is necessary. Yet note that the filter bank illustrated in Equation (5) is oversampled by $3 / 2$, not by 2 . Though, if the filter bank is iterated a single time on its low-pass branch (h0), the total oversampling rate will be 7/4. For a three-stage filter bank, the oversampling rate will be $15 / 8$. When this filter bank is iterated on its lowpass branch indefinitely, the total oversampling rate increases to 2, which is consistent with the redundancy of the frame for L2 (R) [12]. For computing fast discrete DDW transforms consider (W) the following transformation matrix for length-7 [13].

$$
W=\left[\begin{array}{cccccccccccc}
h_{0}(0) & h_{0}(1) & h_{0}(2) & h_{0}(3) & h_{0}(4) & h_{0}(5) & h_{0}(6) & 0 & 0 & \Lambda & 0 & 0 \\
0 & 0 & h_{0}(0) & h_{0}(0) & h_{0}(2) & h_{0}(3) & h_{0}(4) & h_{0}(5) & h_{0}(6) & \Lambda & 0 & 0 \\
\mathrm{M} & \mathrm{M} & \mathrm{M} & \mathrm{M} & \mathrm{M} & \mathrm{M} & \mathrm{M} & \mathrm{M} & \mathrm{M} & \mathrm{M} & \mathrm{M} & \mathrm{M} \\
h_{0}(2) & h_{0}(3) & h_{0}(4) & h_{0}(5) & h_{0}(6) & 0 & 0 & 0 & 0 & \Lambda & h_{0}(0) & h_{0}(1) \\
h_{1}(0) & h_{1}(1) & h_{1}(2) & h_{1}(3) & h_{1}(4) & h_{1}(5) & h_{1}(6) & 0 & 0 & \Lambda & 0 & 0 \\
0 & 0 & h_{1}(0) & h_{1}(1) & h_{1}(2) & h_{1}(3) & h_{1}(4) & h_{1}(5) & h_{1}(6) & \Lambda & 0 & 0 \\
\mathrm{M} & \mathrm{M} & \mathrm{M} & \mathrm{M} & \mathrm{M} & \mathrm{M} & \mathrm{M} & \mathrm{M} & \mathrm{M} & \mathrm{M} & \mathrm{M} & \mathrm{M} \\
h_{1}(2) & h_{1}(3) & h_{1}(4) & h_{1}(5) & h_{1}(6) & 0 & 0 & 0 & 0 & \Lambda & h_{1}(0) & h_{1}(1) \\
h_{2}(0) & h_{2}(1) & h_{2}(2) & h_{2}(3) & h_{2}(4) & 0 & 0 & 0 & 0 & \Lambda & 0 & 0 \\
0 & 0 & h_{2}(0) & h_{2}(1) & h_{2}(2) & h_{2}(3) & h_{2}(4) & 0 & 0 & \Lambda & 0 & 0 \\
\mathrm{M} & \mathrm{M} & \mathrm{M} & \mathrm{M} & \mathrm{M} & \mathrm{M} & \mathrm{M} & \mathrm{M} & \mathrm{M} & \mathrm{M} & \mathrm{M} & \mathrm{M} \\
h_{2}(2) & h_{2}(3) & h_{2}(4) & 0 & 0 & 0 & 0 & 0 & 0 & \Lambda & h_{2}(0) & h_{2}(1)
\end{array}\right]_{\frac{3 N}{2} x N}
$$

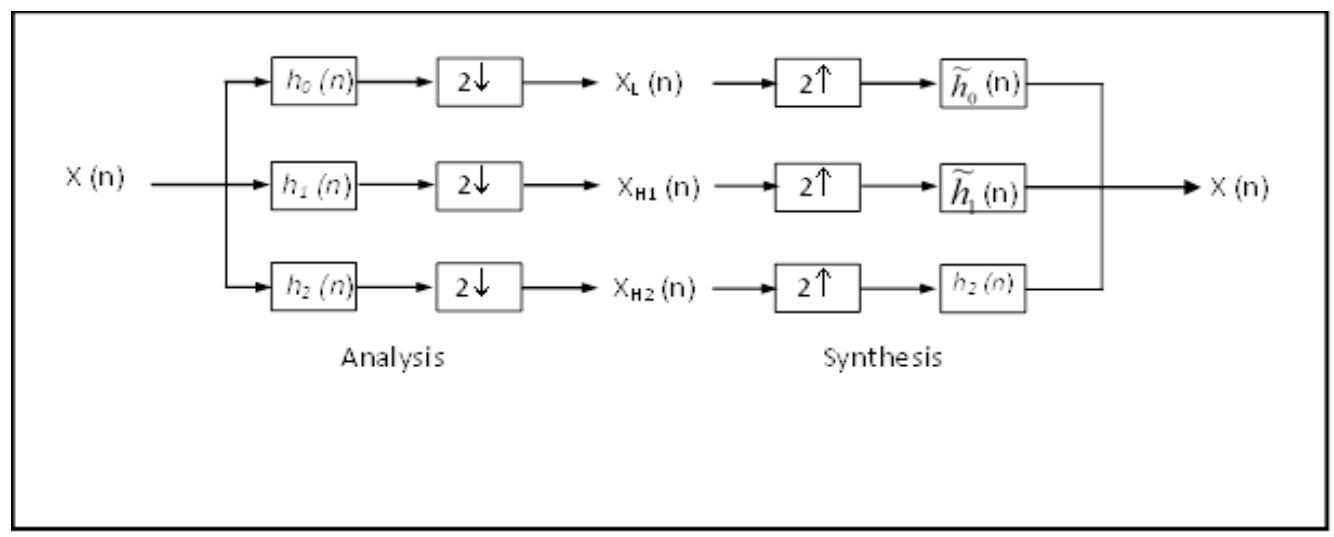

Figure 1: Analysis and Synthesis of 1-D Single Stages for DDWT.

\subsection{Literature Review for Image Compression}

Still, several difficult problems remain unresolved in the Image compression. There are applied to the graphic images algorithms, the basic concepts to store graphic images, colour space, it is also discussed in the summary, proposed a context novel based dual wavelet transform coding approach (CBTC) which combines BWT with highly ranked context account on the basis of coding scheme to compress an integral part of scale of gray images. Nationality [14], the two compression methods for irregular 3D mesh sequence with constant communication using integer wavelet analysis of the exact spatial technique. Also in [15], fractal image compression coding proposed a plan based on wavelet transform with the search of diamonds. In addition, Liu Hui and Silang in [16], proposed a new image coding method based on DWT and quad-tree decomposition. In [17], it was proposed to link new wavelet encoding a large tree way to improve the image compression together within the wavelet tree to facilitate the encryption algorithm. In [18], the new wavelet transform image encryption algorithm with DWT is applied to the original image. Moreover, in [19] reported the design and field programmable 
gate array (FPGA) implementation of system heaviness 2-D DBWT N separable. Zhang et al studied the conversion of the two categories of coding and sub and coding for image compression NDE ultrasonic [20]. In [21], the proposed new 3D image based on wavelet transforms of each of the images and estimate the disparity between the sub band stereo pair compression system. Pantene others, compression techniques made to improve the ultrasound images and the catheter by applying wavelet transform outperforms discrete cosine transform [21]. Image Compressor fast used bi-orthogonal wavelet transform, which gives high-speed calculations and pressure excellent performance worked in [22]. Presentation Angelidis in [23] technique for MR image compression coding based on the conversion plan using a wavelet transform and vector quantization.

\subsection{Optimize DWT Compression Algorithms}

To calculate a single level discrete Wavelet transform for 1-D signal the next steps should be followed: 1. Checking input dimensions: Input vector should be of length $\mathrm{N}$, where $\mathrm{N}$ must be even and $\mathrm{N}$ length (analysis filters).

2. Construct a transformation matrix: Using transformation matrices given in equation (5) .

3. The transformation of the input vector, which can be done by putting on matrix multiplication to the $(3 \mathrm{~N} / 2) \mathrm{xN}$ constructed transformation matrix by the $\mathrm{Nx} 1$ input vector.

$$
Y=[W] \frac{3 N}{2} \times N \cdot[X]_{N \times 1}
$$

Where:

$$
X_{L}=Y\left[0: \frac{N}{2}-1\right], \quad X_{H 1}=Y\left[\frac{N}{2}: N-1\right]
$$

Figure (2) shows the 1-D wavelet Compression, inverse forward transform, algorithms. For a complete DDWT Compression of the Image Xn with $2 n$ entries, the Max Compression Step can be defined in the domain of $[1, \mathrm{n}]$. Compression Step implements one step of the wavelet Compression in an array of data. [12] As we know, images are 2-D signals. A simple way to perform wavelet decomposition on an image is to alternate between operations on the rows and columns. First, wavelet Compression is performed on the pixel values in each row of the image. Then, wavelet Compression is performed to each column of the previous result. The process is repeated to perform the complete wavelet decomposition. 


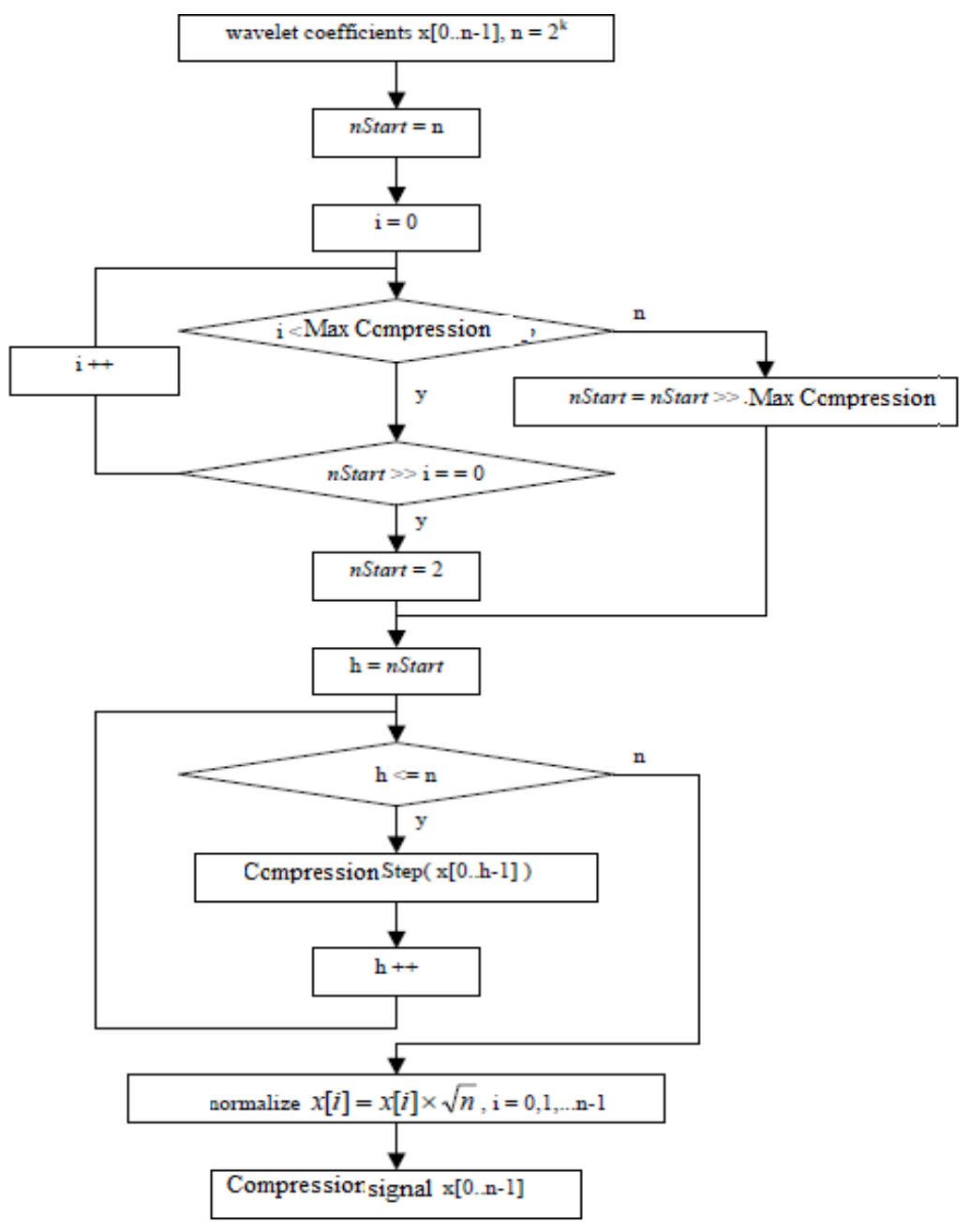

Figure 2: DDWT Composition Algorithm in Min-Image algorithms

\section{PROPOSED THE DDWT TECHNIQUE FOR COMPRESSION 2-D SIGNAL}

\subsection{Computation of the DDWT for 2-D}

As simplifying design complexity is one of the main anxieties of this paper, and depending on the mathematical characteristics of the transformation matrix is given in equation (5), a fast orthogonal-based transform can be determined with the more simplified computation procedure. Starting from the equivalence of two matrix theorem, the equivalence relation $\mathrm{Y}=\mathrm{WXT}$, where $\mathrm{W}$ and $\mathrm{T}$ are non-singular, is a very general one. It can be thought of as a transformation of a matrix $\mathrm{X}$ into a matrix $\mathrm{Y}$, where both are square matrices, equivalent and having the same rank. Many applications involve special cases of equivalence transformations in which $\mathrm{W}$ and $\mathrm{T}$ have further properties. Thus,

$$
\text { If } T=W T=W^{-1}
$$

Then: $Y=W \times X \times W T=W \times X \times W^{-1}$

An orthogonal transformation and $\mathrm{Y}$ is orthogonal to $\mathrm{X}$. So as the transformation matrices are given in (5) transpose are equal to their inverse matrices the orthogonal transformation of ( $W$ ) can be used in the computation of discrete DDWT. 
To compute a single level Orthogonal -based discrete DDWT for 2-D signal the next steps should be followed:

a. Checking input dimensions: Input matrix should be of length $N \times N$, where $N$ must be even and $N>=$ length filters.

b. For a $N \times N$ matrix input 2-D signal $\mathrm{X}$, build a $3 N / 2 \times N$ transformation matrix, $\mathrm{T}$ using transformation matrices is given in equation (5).

c. Apply Transformation by multiplying the transformation matrix by the input matrix by the transpose of the transformation matrix

$[\mathrm{y}]=[\mathrm{W}] \times[\mathrm{X}] \times\left[\mathrm{W}^{\prime}\right]$

This multiplication of the three matrices results in the final discrete DDWT transformed matrix.

3.2 Numerical Cases of Calculating 2D-DDWT.

a. Let $X$ be the input 2-D signal, $X=\operatorname{rand}(8,8)$

$\begin{array}{cccccccc}{[\mathrm{X}]} & 6 \times 8 \\ 0 & 1 & 2 & 3 & 4 & 5 & 6 & 7 \\ 1 & 2 & 3 & 4 & 5 & 6 & 7 & 8 \\ 2 & 3 & 4 & 5 & 6 & 7 & 8 & 9 \\ 3 & 4 & 5 & 6 & 7 & 8 & 9 & 0 \\ 1 & 3 & 6 & 5 & 4 & 3 & 2 & 7 \\ 6 & 5 & 6 & 7 & 7 & 6 & 7 & 4\end{array}$

$\begin{array}{cccccccc}{[\mathrm{X}]} & 8 \times 8= \\ 0 & 1 & 2 & 3 & 4 & 5 & 6 & 7 \\ 1 & 2 & 3 & 4 & 5 & 6 & 7 & 8 \\ 2 & 3 & 4 & 5 & 6 & 7 & 8 & 9 \\ 3 & 4 & 5 & 6 & 7 & 8 & 9 & 0 \\ 1 & 3 & 6 & 5 & 4 & 3 & 2 & 7 \\ 6 & 5 & 6 & 7 & 7 & 6 & 7 & 4 \\ 0 & 0 & 0 & 0 & 0 & 0 & 0 & 0 \\ 0 & 0 & 0 & 0 & 0 & 0 & 0 & 0\end{array}$

b. For an $8 \times 8(\mathrm{~N} \times \mathrm{N}))$ input 2-D signal, $X$, and length (6) filter, constructs a $12 \times 8\left(\frac{3 N}{2} \times N\right)$ transformation matrix, $W$, using length (6) coefficients filter: 


$\begin{array}{cccccccc}{[\mathbf{W}]_{\mathbf{1 2} \times \mathbf{8}}=} & & & & & & & \\ 0.1430 & 0.5174 & 0.6396 & 0.2443 & -0.0755 & -0.0546 & 0 & 0 \\ 0 & 0 & 0.1430 & 0.5174 & 0.6396 & 0.2443 & -0.0755 & 0.0546 \\ -0.0755 & -0.0546 & 0 & 0 & 0.1430 & 0.5174 & 0.6396 & 0.2443 \\ 0.6396 & 0.2443 & -0.0755 & -0.0546 & 0 & 0 & 0.1430 & 0.5174 \\ -0.0185 & -0.0669 & -0.0739 & 0.0004 & 0.5811 & -0.4222 & 0 & 0 \\ 0 & 0 & -0.0185 & -0.0669 & -0.0739 & 0.0004 & 0.5811 & -0.4222 \\ 0.5811 & -0.4222 & 0 & 0 & -0.0185 & -0.0669 & -0.073 & 0.0004 \\ -0.0739 & 0.0004 & 0.5811 & -0.4222 & 0 & 0 & -0.0185 & 0.0669 \\ -0.0460 & -0.1666 & 0.0031 & 0.6776 & -0.4681 & 0 & 0 & 0 \\ 0 & 0 & -0.0460 & -0.1666 & 0.0031 & 0.6776 & -0.4681 & 0 \\ -0.4681 & 0 & 0 & 0 & -0.0460 & -0.1666 & 0.0031 & 0.6776 \\ 0.0031 & 0.6776 & -0.4681 & 0 & 0 & 0 & -0.0460 & 0.1666\end{array}$

c. Apply transformation:

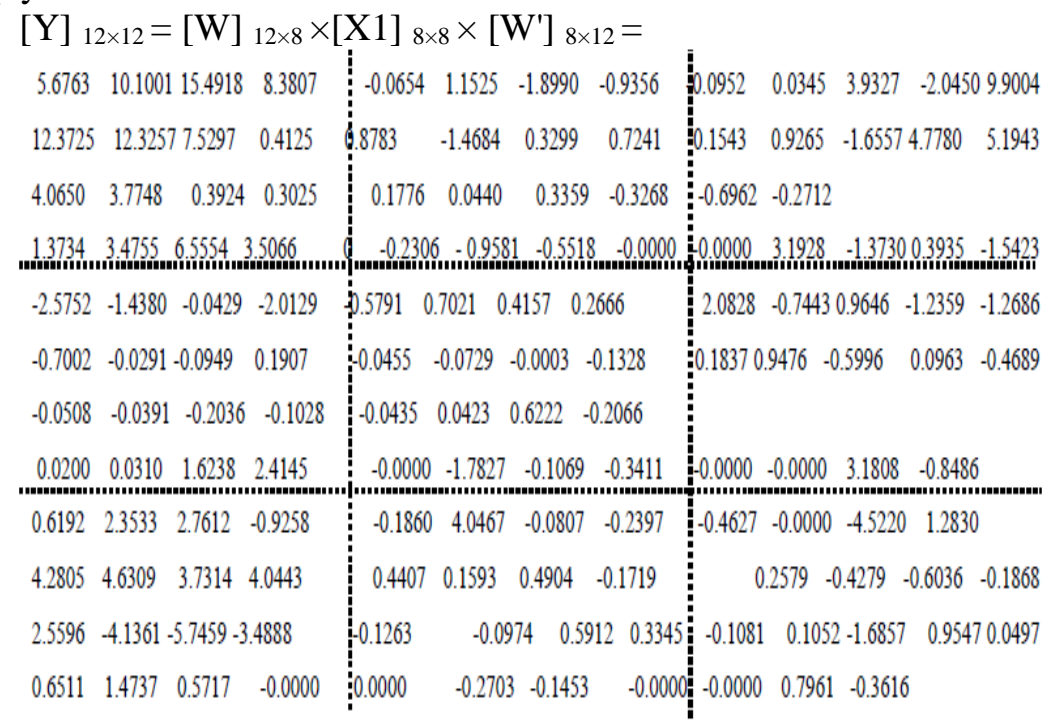

\subsection{Computation of the Inverse DDWT}

To calculate a single level 2-D inverse discrete DDW transforms using non-separable method the next steps should be shadowed:

1. Let $Y$ be the $3 N / 2 \times 3 N / 2$ DD-Wavelet transformed matrix.

2. Construct $N x 3 N / 2$ reconstruction matrix, $T=W^{T}$, with transformation matrices given in equation (5).

3. Reconstruction of the input matrix by multiplying the reconstruction matrix by the input matrix by the transpose of the reconstruction matrix.

To find the 2D inverse DDWT by applying input matrix reconstruction:

$$
[\mathrm{X} 3]_{8 \times 8}=[\mathrm{W}]_{8 \times 12} \times[\mathrm{y}]_{12 \times 12} \times\left[\mathrm{W}^{\prime}\right]_{8 \times 12}
$$




$\begin{array}{llllllll}0.0000 & 1.0000 & 2.0000 & 3.0000 & 4.0000 & 5.0000 & 6.0000 & 7.0000 \\ 1.0000 & 2.0000 & 3.0000 & 4.0000 & 5.0000 & 6.0000 & 7.0000 & 8.0000 \\ 2.0000 & 3.0000 & 4.0000 & 5.0000 & 6.0000 & 7.0000 & 8.0000 & 9.0000 \\ 3.0000 & 4.0000 & 5.0000 & 6.0000 & 7.0000 & 8.0000 & 9.0000 & 0.0000 \\ 1.0000 & 3.0000 & 6.0000 & 5.0000 & 4.0000 & 3.0000 & 2.0000 & 7.0000 \\ 6.0000 & 5.0000 & 6.0000 & 7.0000 & 7.0000 & 6.0000 & 7.0000 & 4.0000 \\ 0.0000 & 0.0000 & 0.0000 & 0.0000 & 0.0000 & 0.0000 & 0.0000 & 0.0000 \\ 0.0000 & 0.0000 & 0.0000 & 0.0000 & 0.0000 & 0.0000 & 0.0000 & 0.0000\end{array}$

At a given level in the iterated filter bank, this separable extension produces nine 2D sub-bands. These sub-bands are shown in Figure (3). Since $L$ is a low-pass filter $\left(h_{0}(n)\right)$ while both $H_{1}$ and $H_{2}$ are high-pass filters $\left(h_{1}(n)\right.$ and $\left.h_{2}(n)\right)$, the $H_{2} H_{2}, H_{2} H_{1}, H_{1} H_{2}, H_{1} H_{1}$ sub-bands each have a frequency-domain support comparable to that of the $H H$ sub-band in a DWT. A similar scheme creates the $H_{l} L, H_{2} L\left(L H_{l}\right.$, $L H_{2}$ ) sub-band with the same frequency-domain support as the corresponding $H L(L H)$ sub-band of the DWT, but with twice as many coefficients. Lastly, note that there is only one sub-band $L L$ with the same frequency-domain support as the $L L$ sub-band in a DWT.

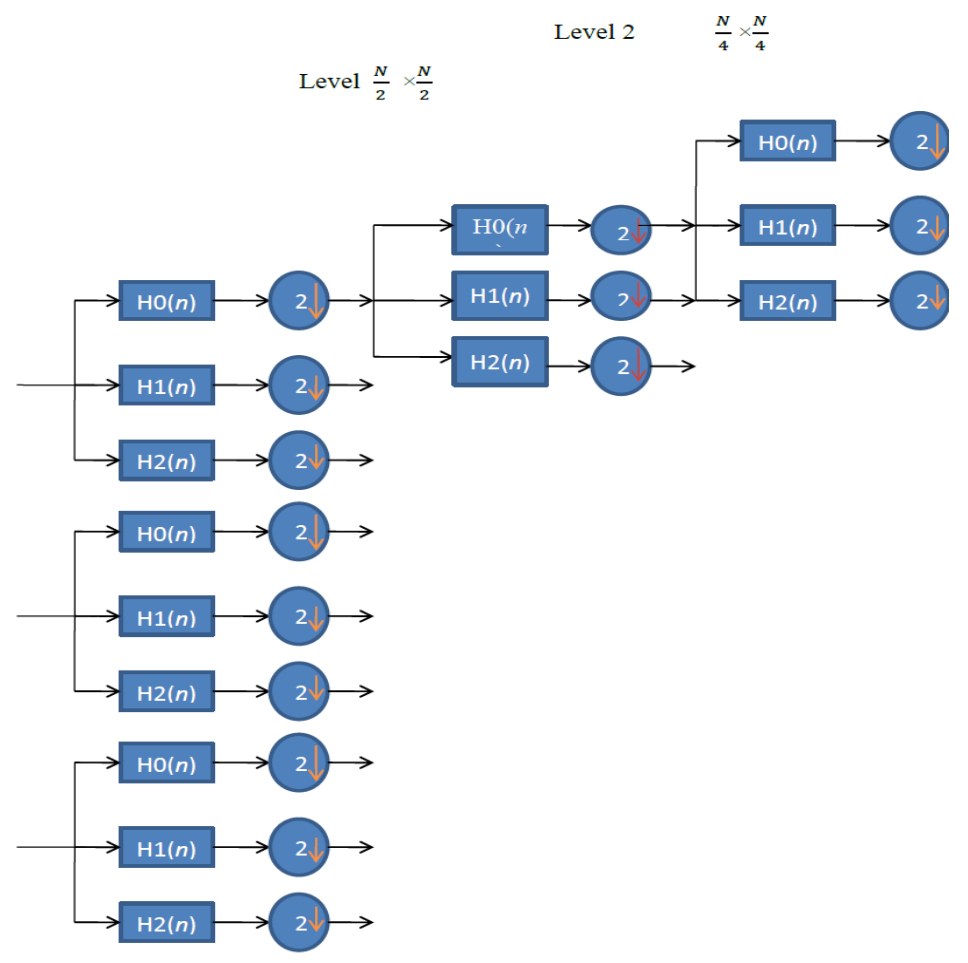

Figure 3: An Oversampled Filter Bank for 2-D Images.

\section{a. Proposed DDWT Image Compression}

There are deliberated the compression from an approximation problem point of view. More specifically, let us fix an orthogonal wavelet $\Psi$. Given an integer $M \geq 1$, it is trying to find the best approximation of $f$ by using a representation:

$\mathrm{F}\{M(x)\}=\sum \operatorname{djk} \Psi j k(x)$

With $M$ non-zero coefficients 
In addition, we are interested in finding an optimal approximation minimizing the RMSE. Because of the orthogonally of the wavelets, this is equivalent to minimizing [24]:

$$
\left\{\sum_{j k}\left|\left(f, \Psi j k(x)-d_{j k}\right)\right|^{2}\right\}^{1 / 2}
$$

The DDWT compression has borrowed some stages in terms of the use of the first stage as an image after an initial conversion, and a simple example will explain in the next section of the DDW transform. Figure (4) shows the image before and after the shift. While spreading the distribution of transactions before the shift, and the vast majority of the wavelet coefficients is small neglect ably.

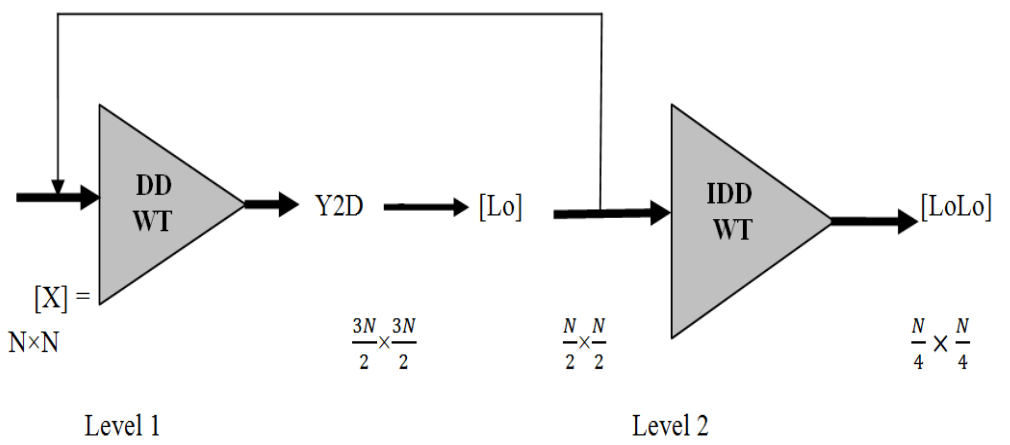

Figure 4: Image compression

Image compression can be achieved users DD- proposed wavelet transform that is low complex using the 2-D signal should be by following these steps:

A. Construct a transformation matrix: Using transformation matrices given in (5) and equation in(8). And, input matrix length $N \times N$, where $N>=$ length filters.

B. Transformation of input rows by applying matrix multiplication to the $(3 N / 2) \times N$ constructed transformation matrix by the $N \times N$ input matrix:

$$
Y 1 D=W * X
$$

C. Apply matrix multiplication to the $(3 N / 2) \times N$ constructed transformation matrix by the $N \times N$ column matrix :

$$
Y 2 D=W * Y 1 D
$$

D. The final discrete DD-Wavelet transformed matrix is equal:

$$
Y 2 D^{\prime \prime}=W * Y 2 D
$$

E. After divided the $Y 2 D$ to nine filters can be selected the $[L L] \mathrm{N} / 6 \mathrm{xN} / 6$

Where, the all details information in LL i.e. image compression to six times less.

F. Can repeat the previous steps to compress the image to 12 times less, however, with less quality

$$
L L 2=W * L 0 L 0
$$

Then, select the first upper part LLLL for all image information as shown in Figure (5) 


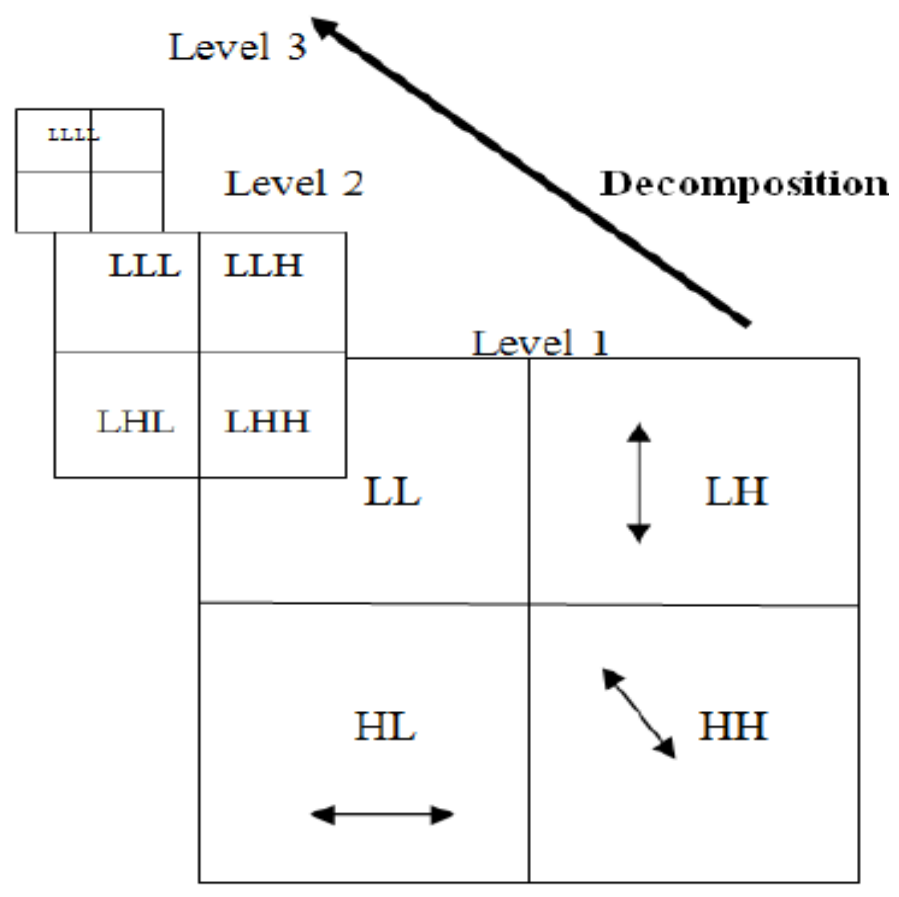

Figure 5: DDWT image compression

\section{SIMULATION RESULTS}

The proposed expansive wavelet transform can be extended to multiple dimensions in a way that is similar to the usually separable implementation. For two-dimensional (2-D) signals, there will be eight sub-bands at each stage: $\mathrm{LB}, \mathrm{LH}, \mathrm{BL}, \mathrm{BB}, \mathrm{BH}, \mathrm{HL}, \mathrm{HB}$, and $\mathrm{HH}$, where "L" stands for "lowpass," H stands for "high-pass," and B stand for "band-pass." These are in addition to the LL sub-band. If the LL sub-band is of size, then the LH sub-band will be of a size, the HH sub-band will be of a size, etc. the relative sizes of each sub-band. In Compression, the critically sampled 2-D DWT has three subbands, LH, HL, HH, each of size. Therefore, the 2-D form of the proposed transform is five-times expansive. That can potentially be substantially less than the 2-D form of the DDWT, which is -times expensive, where is the number of stages as shown in Figure (6). 


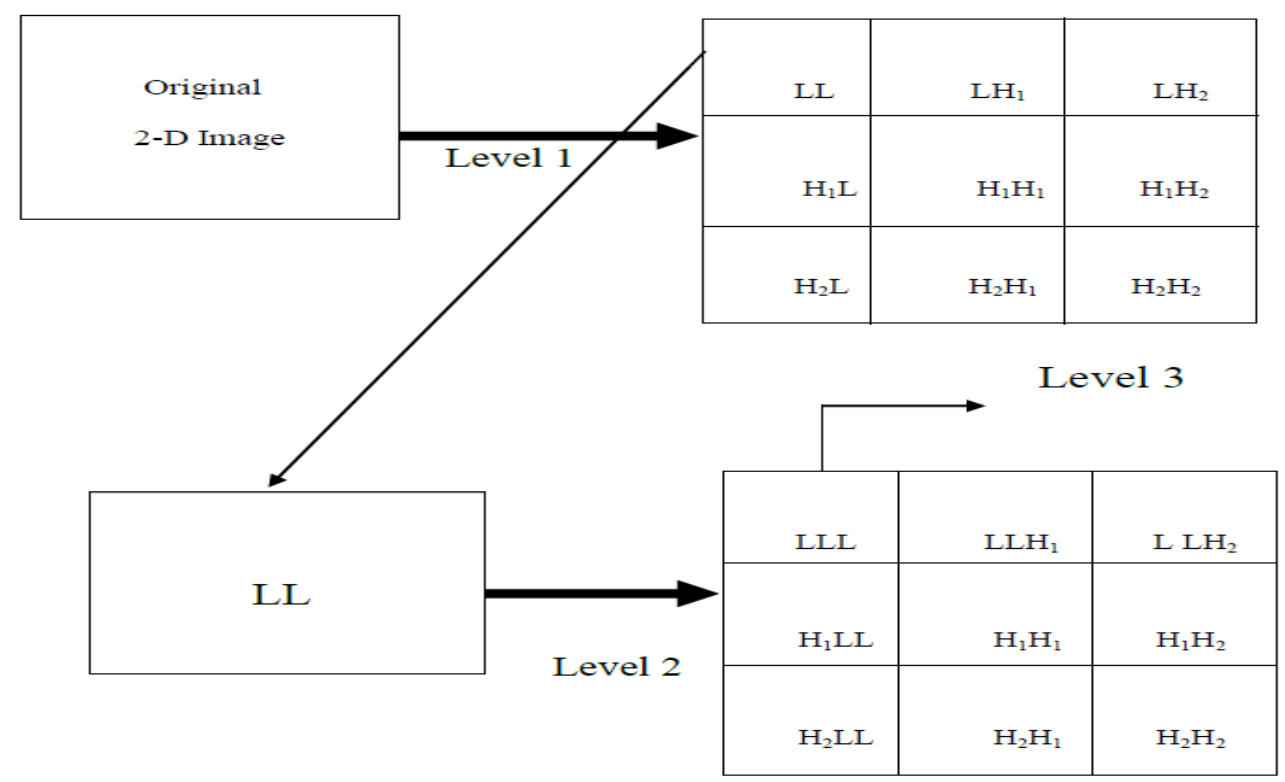

Figure 6: Compressions of Image sub-bands after 2-level decomposition for DDDWT

\subsection{Result DDWT based general Iamge Compression}

Figure (7-a) and Figure (7-b) shown as the Original Fountain's, colour and black and white, Image with size $(550 * 700)$. That equal of dimensions image with adding zeroes, then Image with size $(1024 * 1024)$ shows in figure (7-c) and image in filter (LL) with compressions of image N/2, with size $(240 * 240)$, image in filter (LLL) with compressions of image N/4 with size $\left(1115^{*} 1115\right)$ where are seeing in Figure (7-d) and Figure (7-e). Lastly, return back the image when applied the inverse DDWT as shown in Figure (7-f).

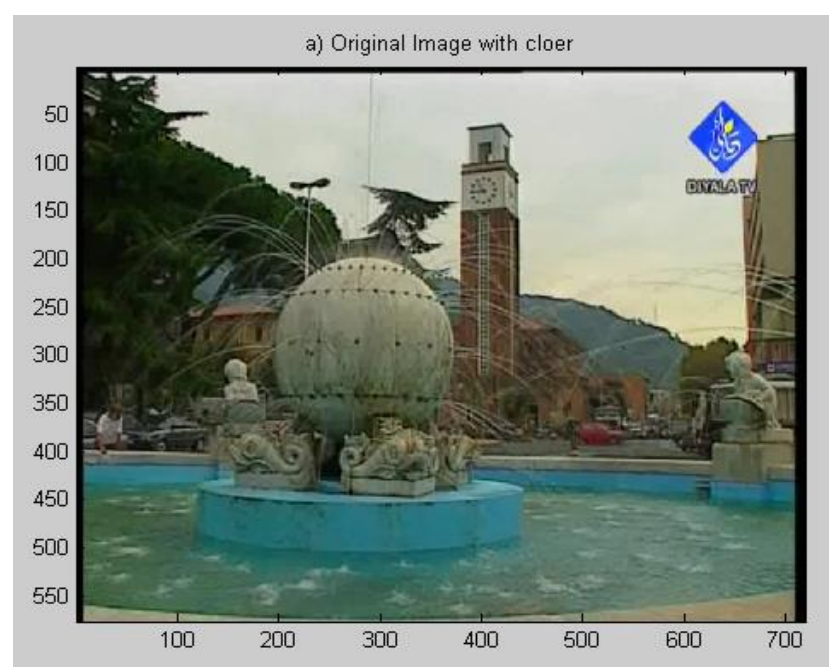

(a) Original image 


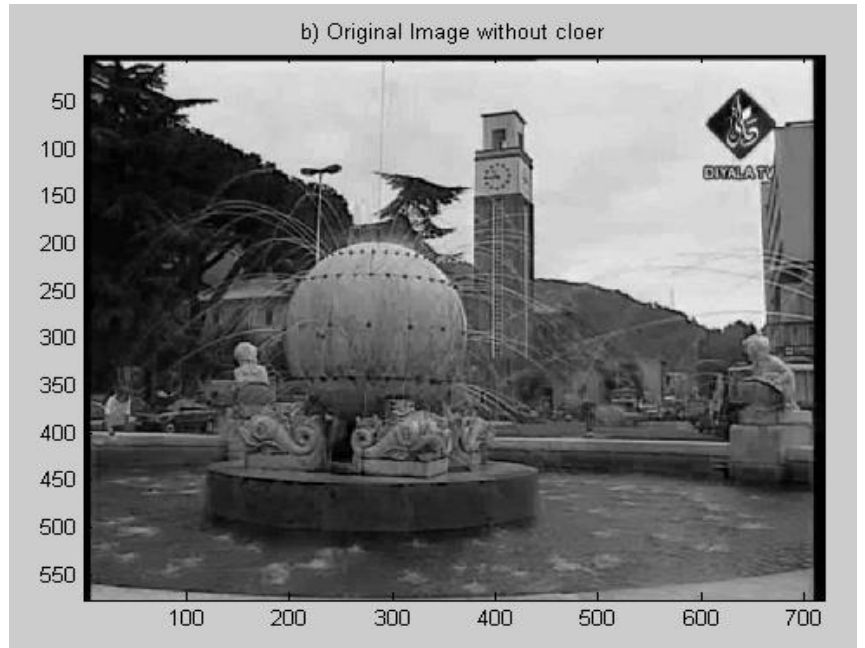

(b) Original image

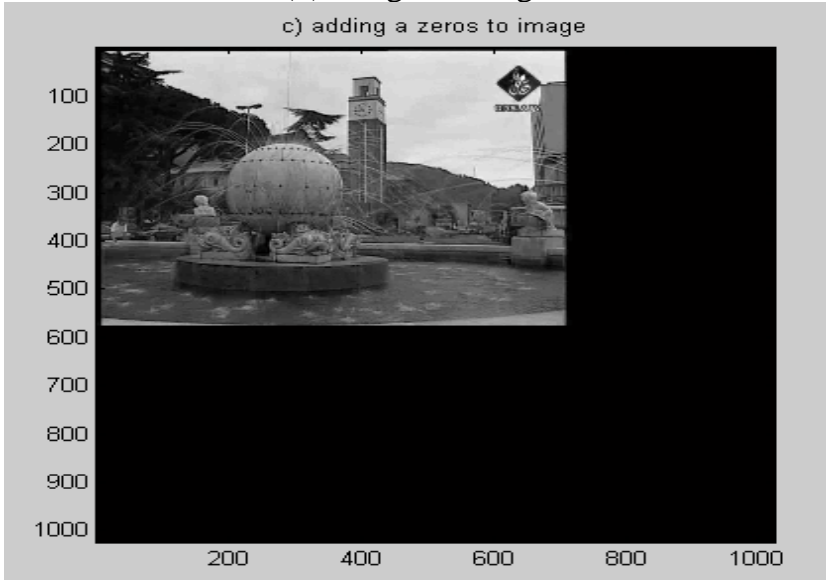

(c) That equal of dimensions image

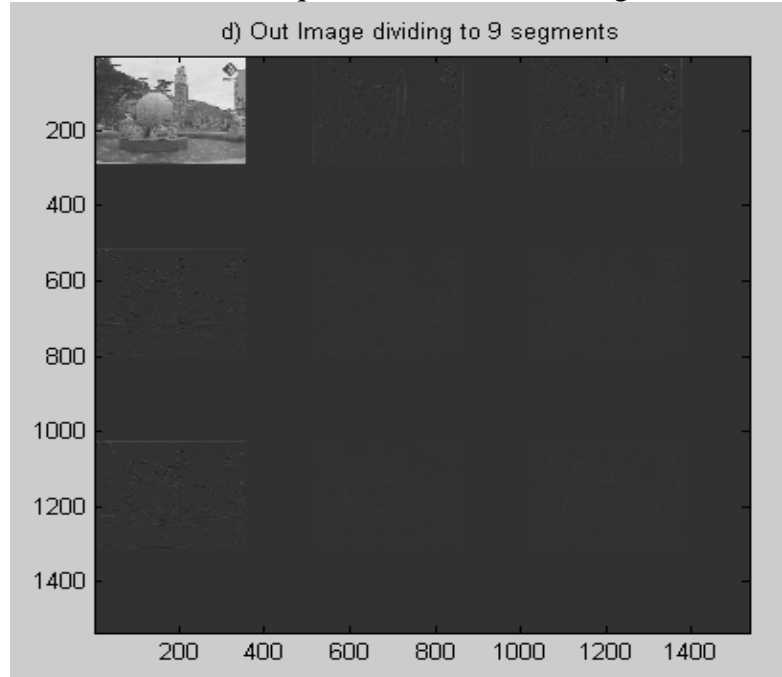

(d) Compression image first level 


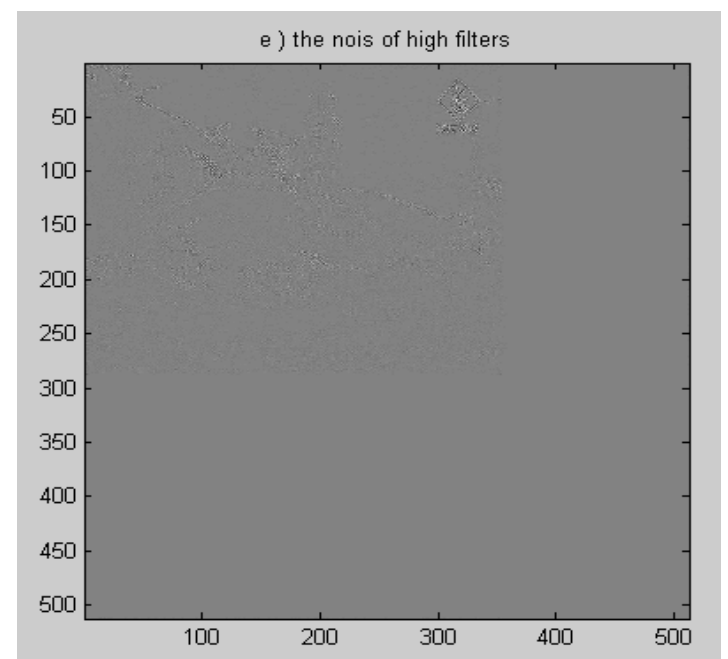

(e) Lossy image

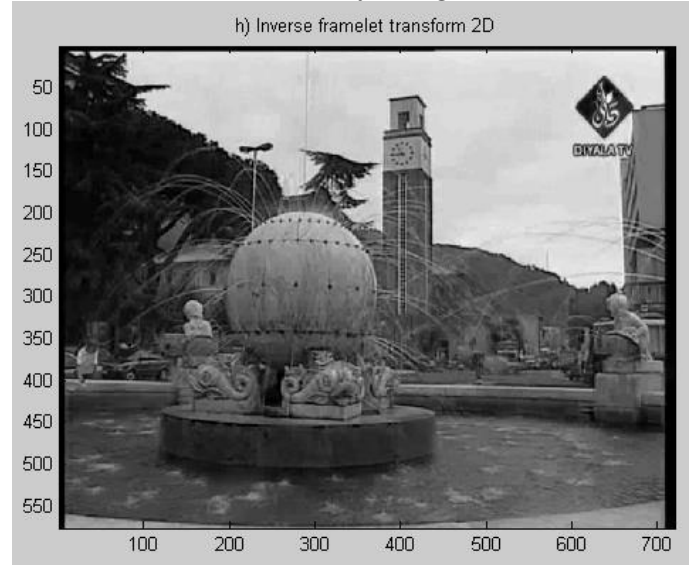

(f) Image inverse DDWT

Figure7: Image Compression based on DDWT

\subsection{Enhancement Medical Image based on DDWT}

Found in some medical fields, usage vast of shapes for image, maybe it was containing the noise. This noise resulting from badness it using of devices, or the devices it is old, or any case, this method found, to clearing how to de-noise image, by using double density wavelet, this layout in Figure (8) is showing stages de-noising as shown in explaining for each figure. In addition, PSNR results of the filtered image with DDWT in Soft Thresholding with 11, for x-ray images as shown in Table 1. 


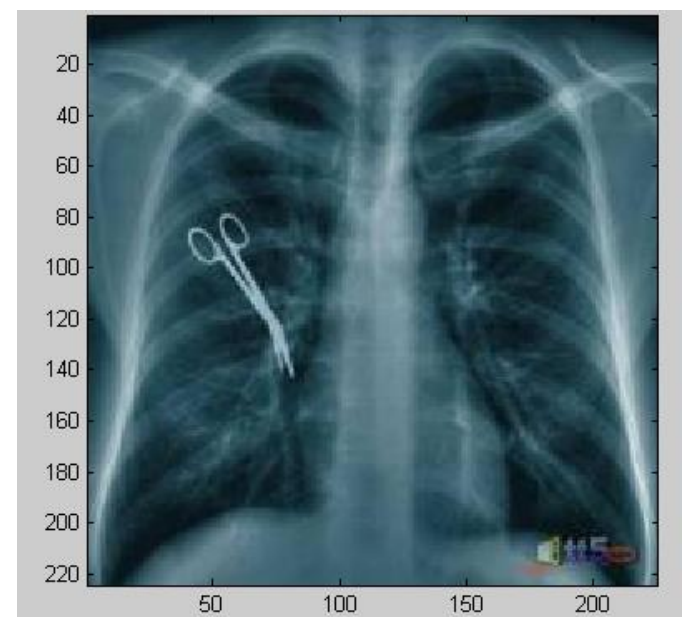

(a) Original $x$-ray image

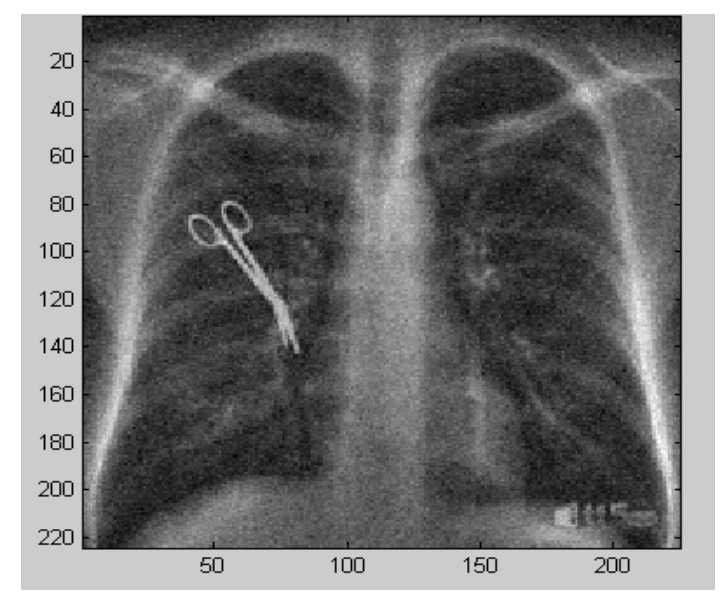

(b) Noisy Gaussian image

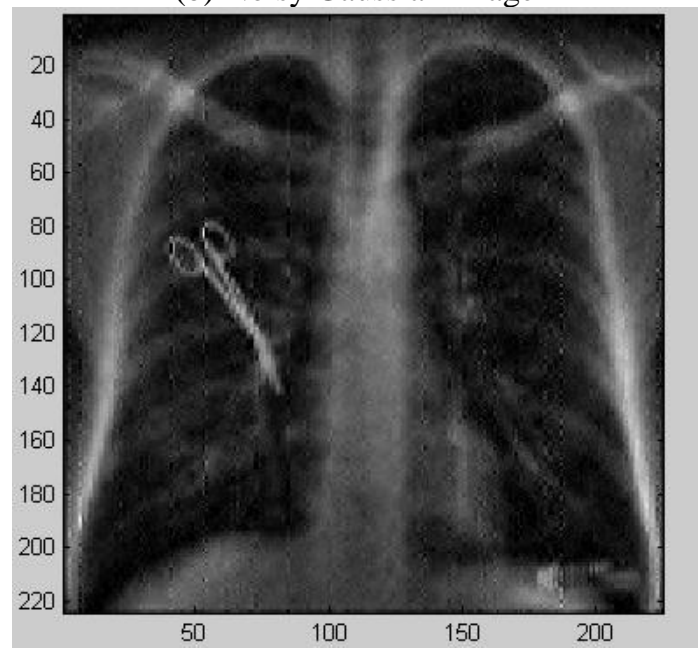

(c) First level improves the image 


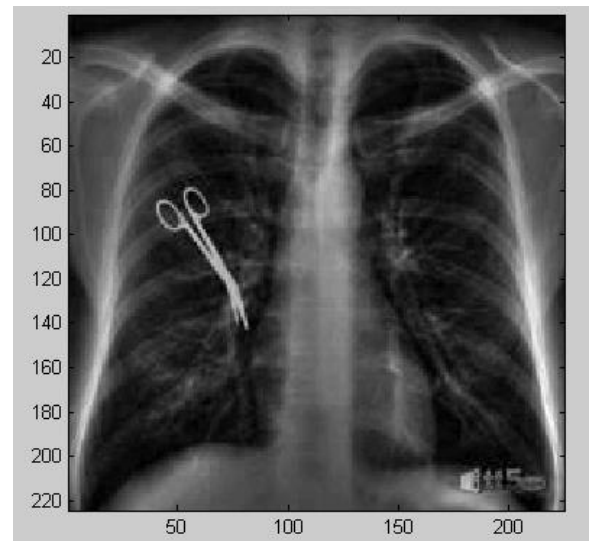

(d) Improve image after inverse DDWT

Figure 8: Improve x-ray images based on DDWT.

Table 1: PSNR Results of the Filtered Three Images

\begin{tabular}{cccc}
\hline Name of image & $\begin{array}{c}\text { PSNR }(\mathrm{dB}) \text { of } \\
\text { Noisy image }\end{array}$ & $\begin{array}{c}\text { PSNR (dB) of De-noising } \\
\text { by DDW }\end{array}$ & $\begin{array}{c}\text { PSNR (dB) of DDWT } \\
\text { after Compressions }\end{array}$ \\
\hline X-ray & 13.55 & 16.69 & 16.04 \\
Haemorrhoids & 12.17 & 17.67 & 16.55 \\
Fountain's & 11.30 & 19.11 & 18.03 \\
\hline
\end{tabular}

\section{CONCLUSIONS}

Discrete Double Density Wavelet Transform plays an important role in image Compression applications because of its double density discrete wavelet transforms. Therefore, its filter divides the image transform into nine levels instead of four levels in DWT. The DDWT is the most commonly used approaches in image de-noising. Therefore, it has a promising future in image enhancement and lifting up the noise types in the field of Framelet transform. This method is a tenderfoot and is better than wavelet transform. It has found applications in Compression medical images as well as in removing low bit rate compression artefacts, the allowance on image. There are two important parameters in DDDWT Compression algorithm, PSNR gives (14.46) value while the PSNR was increased in the proposed compression method, it gives (17.03) PSNR value when the noise variance is (22). Furthermore, MSE equal 2.5896e-14 and, SNR after de-noising DDWT equal $17.03 \mathrm{~dB}$ while PSNR = $15.34 \mathrm{~dB}$ and SNR-Noisy Image $=14.78 \mathrm{~dB}$. A system for image compression using DDW transforms whether for a colour image or the image without colour proved to provide good results. 


\section{REFERENCES}

[1] Ivan W. Selesnick, Member, "Higher Density Discrete Wavelet Transform", IEEE, Transactions On Signal Processing, Vol. 54, NO.8, 2006.

[2] Ali K. Nahar,"A Framele - Based Approach forImage Denoising", Thesis, for the Degree of Master of Science in Electronic Engineering, Department of Electrical and Electronic Engineering, University of Technology, Baghdad - Iraq, July2008.

[3] Rafael C. Gonzalez and Richard E. Woods. Digital Image Processing. Pearson Education, Inc., Upper Saddle River, New Jersey, third edition, 2008.

[4] I.W. Selesnick "Smooth "Wavelet Tight Frames with Zero Moments" Research supported by NSF under CAREER Grant CCR-987452. Applied and Computational Harmonic Analysis, Vol .10, pp:163-18, 2001.

[5] Ali K. Nahar "Color Image Denoising Based on Framelet Transforms" 1st edition, LAP LAMBERT Academic Published, 2015.

[6] W. Selesnick and L. Sendur, "Iterated Oversampled Filter Banks and Wavelet Frames" in Wavelet Applications in Signal and Image Processing VIII, San Diego, 2000.

[7] HN Abduallah, AN Abdallah, A Kareem "A Novel Computing Method for 3D Double Density DWT" J. Eng. Applied Sci Vol. 3 , No. 6 ,446-450, 2008.

[8] M. Victor, P. Alas "Complex Wavelet Transform in Signal and Image Analysis" Institute of Chemical Technology, Department of Computing and Control Engineering.Http://taco.poly.edu/WaveletSoftware, 2004.

[9] P. Chatterjee and P. Milanfar, "Clustering-based Denoising with Locally Learned Dictionaries", IEEE, Transactions on Image Processing, vol. 18, no. 7, pp. 1438-1451, 2009.

[10] P. D. SHUKLA"ComplexWavelet Transforms and Their Applications" A Dissertation Submitted of Signal Processing Division, Department of Electronic and Electrical Engineering University of Strathclyde Scotland United Kingdom, October 2003.

[11] H. N. Al-Taai"A Novel Fast Computing Method for Framelet Coefficients", American Journal of Applied Sciences Vol.5 No.11pp. (1522-1527), 2008.

[12] R. C. Gonzalez and R. E. Woods, "Digital Image Processing"2nd Edn1, Prentice Hall Inc. Upper Saddle River, New Jersey, 2002.

[13] Hadeel N. Abduallah and Ali K. Nahar " Image Denoising Using Framelet Transform" Eng. \& Tech. Journal, University of Technology, Baghdad, Vol 28, No. 13, 2010.

[14] Sandip Mehta, "Medical Image Compression based on ROI using Integer Wavelet Transform ", International Journal on Future Revolution in Computer Science \& Communication Engineering, Vol.: 3 no. 10 p.p $174-180,2017$.

[15] AThirumoorthi C., Karthikeyan T., "A study on discrete wavelet transform compression algorithm for medical images", Biomedical Research; 28 (4): 1574-1580, 2017.

[16] Jovanovic, L., A. Piurica. "Fuzzy logic-based approach to wavelet denoising of 3D images produced by time-of-flight cameras." Optics Express Vol.18, No. 22, pp: 22651- 22676, 2010.

[17] Ali K. Nahar" Data Weighted Averaging (DWA) Technique with 1st order Noise-shaping to Improve 6-bit Digital-to-Analog Convertor (DAC) Performance" Journal of Babylon University/Engineering Sciences, Vol 21, No. 5, 2013.

[18] HilalNaimi, AmelBahaHoudaAdamou-Mitiche, Lahce'neMitiche "Medical image denoising using dual-tree complex thresholding wavelet transform and Wiener filter"Journal of King Saud University - Computer and Information Sciences Vol. 27, No 1, pp:40-45, 2015.

[19] AsmaaAbassAjwad," Noise reduction of ultrasound image using wiener filtering and haar wavelet transform techniques". DiyalaJ.Med. Vol.2 (No.1) 2012.

[20] Chang, S.G., Yu, B., Vetterli, M., 2000. Adaptive wavelet thresholding for image denoising and compression. IEEE Trans. Image Proc. 9 (9), 1532-1546.

[21] Ismail, B., Anjum Khan, 2012. Image de-noising with a new threshold value using wavelets. J. Data Sci. 10, 259-270. 
[22] Kaur, Jaspreet, Kaur, Ranjeet, 2013. Image denoising for speckle noise reduction in ultrasound images using thedwt technique. IJAIEM 2 (6).

[23] Mitiche, Lahce`ne, Adamou-Mitiche, AmelBahaHouda, Naimi, Hilal. "Medical image denoising using Dual Tree Complex ThresholdingWavelet Transform", 2013, IEEE Jordan Conference on Applied Electrical Engineering andComputing Technologies (AEECT), Amman, Jordan, 2013. IEEECatalog Number: CFP1366P-PRT, ISBN: 978-1-4799-2305-2.

[24] Naga Prudhvi Raj, V., Venkateswarlu, T., 2012. Denoising of medicalimages using dual-tree complex wavelet transform. Proc. Technol.4, 238-244, 2012. 